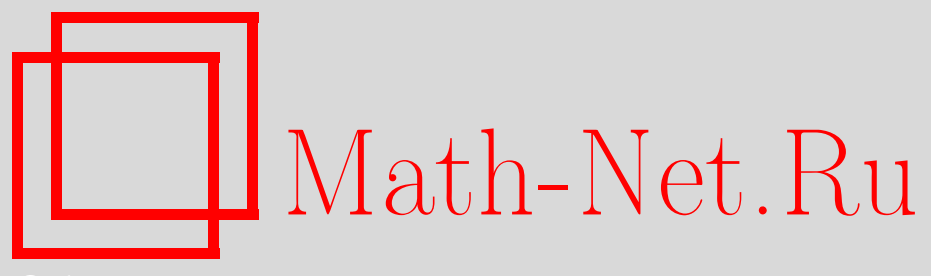

И. Я. Новиков, М. А. Скопина, Почему в разных структурах базисы Хаара одинаковые?, Матем. заметки, 2012, том 91, выпуск 6, 950-953

DOI: https://doi.org/10.4213/mzm9392

Использование Общероссийского математического портала Math-Net.Ru подразумевает, что вы прочитали и согласны с пользовательским соглашением http://www . mathnet.ru/rus/agreement

Параметры загрузки:

IP: 107.22 .136 .117

26 апреля 2023 г., 05:13:32

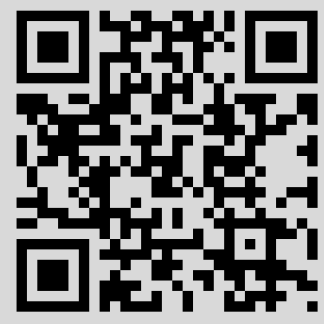




\section{Почему в разных структурах базисы Хаара одинаковые?}

\section{И. Я. Новиков, М. А. Скопина}

Система Хаара [1] является модельным примером базиса всплесков в $L_{2}([0,1])$. Активно изучаться всплески начали в конце прошлого века в связи с созданием теории кратномасштабного анализа (KMA). Вскоре возник интерес к базисам всплесков, определенных на различных структурах. В [2], [3] разработана теория KMA на группе Кантора и найдены различные ортогональные базисы всплесков, существенно отличающиеся от базиса Хаара. Группа 2-адических чисел имеет много сходства с группой Кантора (то же каноническое представление элементов и метрика), различаются они только действием "+", но это приводит к принципиально разным теориям всплесков и КМА. В частности, в [4] доказано, что, в отличие от группы Кантора, не существует $p$-адического КМА с ортогональной масштабирующей функцией, не совпадающего с KMA Хаара. В то же время, p-адический базис Хаара (впервые выписанный в [5]) имеет точно такой же вид, что и базис Хаара на группе Кантора при $p=2$ или Виленкина при $p>2$. В [6] изучены базисы Хаара на отрезке $[0,1]$, у которых на каждом уровне $j$ свой коэффициент сжатия $p_{j}$. В работах [7]-[10] построены базисы Хаара на кольцах целых $p$-адических чисел и проективных пределах конечных циклических групп в одномерном и многомерном случае. В [8; замечание 2.4] отмечается наличие сходства базиса Хаара на нульмерной группе и базиса Хаара на $[0,1]$ в [6]. В настоящей заметке мы даем простое объяснение этого сходства, показывая, что все упомянутые выше базисы Хаара могут быть определены одними и теми же формулами. Будет описана достаточно общая схема построения базисов Хаара, при этом не требуется даже наличие группового действия "+" (его использование только создает некоторое удобство для записи функций Хаара, но можно обойтись и без этого). Нужно только наличие "не слишком плохой" топологии в пространстве с мерой и подходящая последовательность разбиений пространства. При этом процесс построения системы Хаара и доказательство ее базисности точно такие же, как для классического базиса Хаара.

Пусть $\mu$ - мера в $\Omega, \Sigma-\sigma$-алгебра измеримых множеств, $\mathbf{p}=\left\{p_{j}\right\}_{j=-\infty}^{\infty}-$ последовательность целых чисел, $p_{j}>1$. Предположим, что существуют наборы множеств $\Omega_{j n}$, $n \in \mathbb{Z}_{+}$, попарно дизъюнктных при каждом $j \in \mathbb{Z}$, такие, что $\mu \Omega_{0 n}=1$ для любого $n \in \mathbb{Z}_{+}$, $\Omega=\bigcup_{n} \Omega_{j n}$ для любого $j \in \mathbb{Z}$, каждое $\Omega_{j-1, n}$ разбивается на $p_{j}$ равновеликих подмножеств $\Omega_{j, n_{k}}, n_{k}=n_{k}(n, j) \in \mathbb{Z}_{+}$. Семейство пар $(j, n)$, нумерующих множества $\Omega_{j n}$, обозначим через $I$ и совокупность $\left\{\Omega_{j n}\right\}_{(j, n) \in I}$ назовем $(H, \mathbf{p})$-разбиением, а ее элементы - ячейками. Обозначим через $\phi_{j n}$ характеристическую функцию множества $\Omega_{j n}$ с нормирующим в $L_{2}(\Omega)$ множителем и положим $V_{j}:=\overline{\operatorname{span}\left\{\phi_{j n}, n \in \mathbb{Z}\right\}}$.

Теорема. Пусть пространство с мерой $(\Omega, \Sigma, \mu)$ снабжено топологией $\tau$ такой, что $\Sigma$ содержит все открытые множества и мера $\mu$ регулярна, $\Omega=\left\{\Omega_{j n}\right\}_{(j, n) \in I}-(H, \mathbf{p})$-разбиение. Если для любого $x \in \Omega$ и любой окрестности $U$ точки $x$ существует ячейка $\Omega_{j n}$, содержащая $x$ и содержащаяся в $U$, то пространства $V_{j}, j \in \mathbb{Z}$, образуют $\mathrm{KMA}_{\text {в }} L_{2}(\Omega)$, т.е. удовлетворяют следуюшим условиям:

(i) $V_{j-1} \subset V_{j}$;

(ii) $\bigcap_{j \in \mathbb{Z}} V_{j}=\{0\}$;

(iii) $\overline{\bigcup_{j \in \mathbb{Z}} V_{j}}=L_{2}(\Omega)$;

(iv) $\left\{\phi_{j n}\right\}_{n \in \mathbb{Z}_{+}}-$ортонормированный базис в $V_{j}$.

Работы первого автора выполнена при поддержке Российского фонда фундаментальных исследований (гранты №№ 11-01-00614, 12-01-00216); работа второго автора выполнена при поддержке Российского фонда фундаментальных исследований (грант № 12-01-00216) и Санкт-Петербургского государственного университета (НИР № 9.38.62.2012). 
ДоказАтельство. Свойства (i) и (iv) очевидны.

Для доказательства (ii) заметим, что функция $f \in \bigcap_{j \in \mathbb{Z}} V_{j}$ должна быть константой на любой ячейке $\Omega_{j n}$. Пусть $f=c$ на $\Omega_{j, n_{0}}$ для некоторого $n_{0}$. Тогда $f=c$ на любой ячейке, содержащей $\Omega_{j n_{0}}$, а значит, и на их объединении, которое имеет бесконечную меру. Откуда, учитывая, что $f \in L_{2}(\Omega)$, получаем $c=0$, что влечет $f=0$ ввиду произвольности $n_{0}$.

Свойство (iii) эквивалентно следующему: для любого множества $A \in \Sigma$ конечной меры и для любого $\varepsilon>0$ существует $I_{1} \subset I$ такое, что

$$
\mu\left(A \Delta \bigcup_{(j, k) \in I_{1}} \Omega_{j k}\right)<\varepsilon .
$$

Это утверждение легко получить с помощью рассуждений, аналогичных приведенным в [11; замечание 10.b.3]. Пусть $A \in \Sigma, \mu A<\infty, \varepsilon>0$. Из регулярности меры следует существование открытого множества $G$ такого, что $A \subset G, \mu(G \backslash A)<\epsilon$. Покажем, что $G$ представимо в виде объединения попарно дизъюнктных ячеек. Обозначим через $\Delta_{0}$ объединение всех ячеек $\Omega_{0 n}$ содержащихся в $G$, через $\Delta_{1}$ - объединение всех ячеек $\Omega_{1 n}$ содержащихся в $G$ и не содержащихся в $\Delta_{0}$. Продолжая аналогично, получим последовательность попарно дизъюнктных множеств $\Delta_{j}, j=0,1, \ldots$, содержащихся в $G$. Положив $\Delta=\bigcup_{j=0}^{\infty} \Delta_{j}$, имеем $\Delta \subset G$. Так как для произвольного $x \in G$ по условию существует ячейка $\Omega_{j n}$, содержащая $x$ и содержащаяся в $G$, то $\Delta=G$. Осталось заметить, что $\mu\left(\Delta \backslash \bigcup_{j=0}^{N} \Delta_{j}\right)<\epsilon$ при достаточно больших $N$.

ЗАмечАниЕ 1 . Если в условиях теоремы топология $\tau$ определяется метрикой, то мера $\mu$ регулярна (см., например, [15]). В этом случае дополнительное предположение о ячейках будет выполнено, если для каждого $x \in \Omega$ диаметры вложенных ячеек, содержащих $x$, стремятся к нулю. В случае метрического пространства и ограниченной последовательности $\left\{p_{j}\right\}_{j \in \mathbb{Z}}$ теорема доказана в [12; теорема 5.1].

Под системой Хаара, порожденной КМА Хаара, мы будем понимать следующую ортонормированную систему в $L_{2}(\Omega)$. Для произвольной пары $(j, n) \in I$ рассмотрим подпространство $W_{j n}$, порожденное характеристическими функциями ячеек $\Omega_{j+1, n_{k}}, k=1, \ldots$, $p_{j+1}$, составляющих разбиение $\Omega_{j n}$, причем функции этого подпространства имеют нулевое среднее. Ясно, что $\operatorname{dim} W_{j n}=p_{j+1}-1$ и члены любого ортонормированного базиса в $W_{j n}$ имеют вид

$$
\psi_{j n}^{\nu}=C_{j+1} \sum_{k=1}^{p_{j+1}} h_{\nu, k} \chi_{\Omega_{j+1, n_{k}}},
$$

где $h_{\nu k}, \nu, k=1, \ldots, p_{j+1}$ - элементы унитарной матрицы (вообще говоря, своей для каждого $(j, n) \in I)$, у которой первая строка состоит из одинаковых элементов. Чаще всего в качестве таких унитарных матриц берут матрицу корней из единицы. Систему Хаара определим как $\Psi:=\bigcup_{(j, n) \in I} \Psi_{j n}$, где $\Psi_{j n}:=\left\{\psi_{j n}^{\nu}\right\}_{\nu=2}^{p_{j+1}}-$ произвольный ортонормированный базис в $W_{j n}$. Если существует оператор $D: \Omega \rightarrow \Omega$ такой, что $D \Omega_{j n}=\Omega_{j+1, n}$ для всех $n \in \mathbb{Z}_{+}$, то, задав базисы $\Psi_{0, k}$, мы имеем систему Хаара традиционного вида

$$
\psi_{j k}^{\nu}(x)=C_{j} \psi_{0 k}^{\nu}\left(D^{j} x\right), \quad k \in \mathbb{Z}_{+}, \quad j \in \mathbb{Z}, \quad \nu=1, \ldots, p_{j+1}-1 .
$$

ЗАмечание 2. Случай $\mu \Omega<\infty$ (можно считать $\mu \Omega=1$ ) совершенно аналогичен: в $(H, \mathbf{p})$-разбиении рассматриваются только ячейки, содержащиеся в $\Omega=\Omega_{00}$, и в систему Хаара добавляется тождественная единица.

Примеры. Пусть $p_{j}=p, p$ простое, $\Omega=\mathbb{Q}_{p}$ - поле $p$-адических чисел, $\mu$ - мера Хаара в $\mathbb{Q}_{p}, D: x \rightarrow p x, \Omega_{0 n}=a_{n}+\mathbb{Z}_{p}, a_{n} \in J$, где $\mathbb{Z}_{p}$ - множество $p$-адических целых, $J=$ $\left\{a_{n}\right\}_{n=0}^{\infty}-$ множество $p$-адических чисел, совпадающих со своей дробной частью, $\Omega_{j n}=$ $D^{j} \Omega_{0 n}$. В этом случае мы получаем базис, построенный в работе [5]. Простейший многомерный базис Хаара можно построить, взяв

$$
\Omega=\mathbb{Q}_{p}^{d}, \quad \Omega_{0 n}=a_{n}+\mathbb{Z}_{p}^{d}, \quad a_{n} \in J^{d},
$$


$D$ - матрица размера $p \times p$, в правом верхнем углу которой стоит $p$, под каждым элементом главной диагонали -1 , а остальные нули. Каждое $\Omega_{0 n}$ разбивается на $p$ равновеликих подмножеств $\Omega_{k}^{n}$, состоящих из векторов $x \in \Omega_{0 n}$ таких, что

$$
x_{1}-\left(a_{n}\right)_{1} \equiv k(\bmod p), \quad k=0,1, \ldots, p-1 .
$$

Нетрудно видеть, что $D^{-1} \Omega_{k}^{n}=\Omega_{0 m}$, где $m=m(n, k) \in \mathbb{Z}_{+}$, и $D$ устанавливает взаимно однозначное соответствие между наборами множеств $\left\{\Omega_{0 n}\right\}$ и $\left\{\Omega_{k}^{n}\right\}$. Положив $\Omega_{j n}=$ $D^{j} \Omega_{0 n}, j \in \mathbb{Z}$, получим $(H, \mathbf{p})$-разбиение $\left\{\Omega_{j n}\right\}_{j \in \mathbb{Z}, n \in \mathbb{Z}_{+}}$. Соответствующие базисы Хаара строились в [13], а сужения базисных функций на $\mathbb{Z}_{p}$ (которые образуют базис в $L_{2}\left(\mathbb{Z}_{p}\right)$, поскольку носитель каждого элемента построенного базиса Хаара либо содержится в $\mathbb{Z}_{p}$, либо не пересекается с $\mathbb{Z}_{p}$ ) рассматривались в [9] с использованием теории нульмерных групп.

Пусть $p_{j}=2, \Omega=C$ - группа Кантора, состоящая из элементов $x=\left\{x_{k}\right\}_{k=-\infty}^{\infty}$, где $x_{k} \in\{0,1\}$, причем $x_{k} \neq 0$ лишь для конечного числа отрицательных $k, D-$ оператор, сопоставляющий каждому $x=\left\{x_{k}\right\}_{k=-\infty}^{\infty}$ элемент $\left\{x_{k-1}\right\}_{k=-\infty}^{\infty}, \mu-$ мера Хаара в $C$. Топология в $C$ определяется нормой $\|x\|=2^{m}$, где $m-$ наименьшее целое для которого $x_{m} \neq 0$. Каждому неотрицательному целому числу

$$
n=2^{m} y_{m}+\cdots+2 y_{1}+y_{0}, \quad y_{k} \in\{0,1\},
$$

сопоставим множество $\Omega_{0 n}$, состоящее из элементов $x$ таких, что $x_{0}=y_{0}, \ldots, x_{-m}=y_{m}$, $x_{k}=0$ при $k<-m$. Положив $\Omega_{j n}=D^{j} \Omega_{0 n}$, имеем $(H, \mathbf{p})$-разбиение и базис Хаара, построенный в [3]. Аналогично строится базис Хаара на группе Виленкина [14].

Пусть $G$ - компактная нульмерная группа, порожденная односторонней цепочкой вложенных подгрупп $G=G_{0} \supset G_{1} \supset G_{2} \supset \cdots$, определяющих топологию. Группа ассоциирована с некоторым набором простых чисел $\left\{p_{j}\right\}_{j=1}^{\infty}$, элементы группы представимы в виде $x=\sum_{k=1}^{\infty} x_{k} g_{k}$, где $x_{k} \in\left\{0,1, \ldots, p_{k}-1\right\}$, а элементы $g_{k} \in G_{k-1} \backslash G_{k}$ удовлетворяют условию $p_{j} g_{j}=g_{j+1}$. Равенства

$$
\mu G_{0}=1, \quad \mu G_{j}=\left(p_{1} \cdots p_{j}\right)^{-1}, \quad j \in \mathbb{N},
$$

определяют меру, инвариантную относительно сдвига, на полукольце всевозможных сдвигов множеств $G_{j}$. Эта мера распространяется на $\sigma$-алгебру $\Sigma$ по схеме Каратеодори. Нетрудно проверить, что эта мера регулярна. Пусть $\Omega=\Omega_{0,0}=G,\left\{\left(y_{j, k, 1}, \ldots, y_{j, k, j}\right)\right\}_{k=0}^{p_{1} \cdots p_{j}-1}$ - занумерованные в произвольном порядке элементы декартового произведения

$$
\left\{0,1, \ldots, p_{1}-1\right\} \times \cdots \times\left\{0,1, \ldots, p_{j}-1\right\} .
$$

Обозначая

$$
\Omega_{j k}=\left\{x: x_{l}=y_{j, k, l}, l=1, \ldots, j\right\}, \quad k=0, \ldots, p_{1} \cdots p_{j}-1, \quad j \in \mathbb{Z}_{+},
$$

получим $(H, \mathbf{p})$-разбиение. Соответствующий базис Хаара строился в [7].

Теперь покажем, как определить $(H, \mathbf{p})$-разбиение в $G^{d}$, порождающее систему Хаара, построению которой посвящена работа [8]. Ограничимся случаем $d=2$. Положим $\Omega=G^{2}$, $\mathbf{p}=\left\{p_{1}, p_{1}, p_{2}, p_{2}, p_{3}, p_{3}, \ldots\right\}$. Каждое $x \in \Omega$ представимо в виде $x=\left(a g_{1}, b g_{1}\right)+y$, где $y \in G_{1}^{2}, a, b \in\left\{0,1, \ldots, p_{1}-1\right\}$. Множество векторов $(a, b)$ образует группу относительно сложения по модулю $\left(p_{1}, p_{1}\right)$, которая распадается на $p_{1}$ циклических подгрупп

$$
\gamma_{k}=\left\{\left(a_{k}, b_{k}\right)+\left(l a^{*}, l b^{*}\right), l=0,1, \ldots, p_{1}-1\right\}
$$

где $\left(a^{*}, b^{*}\right)$ - любой ненулевой элемент группы. Ячейку $\Omega_{1 k}$ определим, включив в нее все $x$, у которых $(a, b) \in \gamma_{k}$. Далее, фиксируя $l$, определим соответствующие ячейки $\Omega_{2, k_{l}}$, составляющие разбиение $\Omega_{1 k}$. Аналогично каждое $\Omega_{2 n}$ сначала разбиваем на $p_{2}$ ячеек $\Omega_{3, n_{k}}$, каждая из которых затем разбивается на $p_{2}$ ячеек $\Omega_{4, k_{l}}$. 


\section{СПИСОК ЦИТИРОВАННОЙ ЛИТЕРАТУРЫ}

[1] А. Haar, Math. Ann., 69:3 (1910), 331-371. [2] В. Ю. Протасов, Ю.А. Фарков, Матем. сб., 197:10 (2006), 129-160. [3] W. C. Lang, SIAM J. Math. Anal., 27:1 (1996), 305-312. [4] S. Albeverio, S. Evdokimov, M. Skopina, J. Fourier Anal. Appl., 16:5 (2010), 693-714. [5] С. В. Козырев, Изв. РАН. Сер. матем., 66:2 (2002), 149-158. [6] Б. И. Голубов, Сиб. матем. журн., 9 (1968), 297-314. [7] С. Ф. Лукомский, Изв. Сарат. ун-та. Нов. сер. Сер. Математика. Механика. Информатика, 9:1 (2009), 14-19. [8] S. F. Lukomskii, Cent. Eur. J. Math., 9:3 (2011), 627-639. [9] С.Ф. Лукомский, Матем. заметки, 90:4 (2011), 541-557. [10] С. Ф. Лукомский, Матем. сб., 201:5 (2010), 41-64. [11] I. Novikov, E. Semenov, Haar Series and Linear Operators, Math. Appl., 367, Kluwer Acad. Publ., Dordrecht, 1997. [12] H. Aimar, A. Bernardis, B. Iaffei, J. Approx. Theory, 148:1 (2007), 12-34. [13] S. Albeverio, S. V. Kozyrev, P-Adic Numbers Ultrametric Anal. Appl., 2:4 (2010), 265-277. [14] Yu. A. Farkov, Facta Univers. Ser.: Elec. Energ., 21:3 (2008), 309-325. [15] Б. М. Макаров, А.Н. Подкорытов, Лекции по вещественному анализу, БХВ-Петербургб, СПб., 2011.

\section{И. Я. Новиков}

Воронежский государственный университет

E-mail: igor.nvkv@gmail.com

\section{М. А. Скопина}

Санкт-Петербургский государственный университет

E-mail: skopina@MS1167.spb.edu
Поступило

04.12.2011 